\title{
FACTORIZATION RESULTS FOR LEFT POLYNOMIALS IN SOME ASSOCIATIVE REAL ALGEBRAS: STATE OF THE ART, APPLICATIONS, AND OPEN QUESTIONS
}

\author{
ZIJIA LI, DANIEL F. SCHARLER, AND HANS-PETER SCHRÖCKER
}

\begin{abstract}
We discuss existence of factorizations with linear factors for (left) polynomials over certain associative real involutive algebras, most notably over Clifford algebras. Because of their relevance to kinematics and mechanism science, we put particular emphasis on factorization results for quaternion, dual quaternion and split quaternion polynomials. A general algorithm ensures existence of a factorization for generic polynomials over division rings but we also consider factorizations for non-division rings. We explain the current state of the art, present some new results and provide examples and counter examples.
\end{abstract}

\section{INTRODUCTION}

The factorization theory of polynomials over division rings has been developed half a century ago in $[1,2]$. It gained new attention in recent years because relations to mechanism science were unveiled $[3,4,5,6,7,8,9,10,11]$. Quaternion polynomials parameterize rational spherical motions. For describing motions in $\mathrm{SE}(2)$ or $\mathrm{SE}(3)$ dual quaternion polynomials are necessary. Their factorization theory turned out to be more complicated and, arguably, more interesting as well.

In this contribution we summarize the current state of the art in the factorization theory of dual quaternion polynomials but we also demonstrate that many results hold for polynomials over certain more general finite-dimensional associative real algebras, most notably finite-dimensional Clifford algebras. Throughout this paper we illustrate the general theory by three prototypical examples with significantly different properties: The quaternions $\mathbb{H}$, the dual quaternions $\mathbb{D H}$, and the split quaternions $\mathbb{S}$ that can model planar hyperbolic kinematics. A fundamental factorization algorithm, based on the factorization of real polynomials, works for generic polynomials over these algebras.

In Section 2 we recall some general results on the factorization of polynomials over rings, in Section 3 we present theoretical and algorithmic results (Theorem 3 and Algorithm 2) on polynomial factorization over quaternions. This is followed by some factorization examples that illustrate the intricacies of polynomial factorization over skew rings (Section 4). There exist polynomials with no, many or even infinitely many factorizations. Some of these factorizations can be computed by means of Algorithm 2 - even if its general applicability is limited to division algebras. Section 5 explains relations of polynomial factorization over quaternion rings to kinematics and mechanism science while Section 6 features a collection of known and new results that allow to compute factorizations or to at least guarantee their existence. The new results of this part include statements on factorizability of quadratic split quaternion polynomials or unbounded motion polynomials.

Date: September 28, 2018.

2010 Mathematics Subject Classification. 12D05, 15A66, 16S36, 30C15, $70 \mathrm{~B} 10$. 


\section{Polynomial Factorization over Rings}

Consider a possibly non-commutative ring $R$ and a polynomial $C=\sum_{i=0}^{d} c_{i} t^{i}$ in one indeterminate $t$ with coefficients $c_{0}, c_{1}, \ldots, c_{d} \in R$. We define the product of two polynomials $A=\sum_{i=0}^{d} a_{i} t^{i}$ and $B=\sum_{i=0}^{e} b_{i} t^{i}$ as

$$
A B:=\sum_{i=0}^{d+e} c_{i} t^{i} \quad \text { where } \quad c_{i}=\sum_{j+k=i} a_{j} b_{k} .
$$

This is really just one possible multiplication rule among others [12]. It is suitable for our purpose because in applications the indeterminate $t$ typically serves as a real parameter and $R$ is an associative real algebra.

We consistently write coefficients to the left of the indeterminate and hence speak of left polynomials. With addition defined in the usual way as $A+B:=\sum_{i=0}^{\max \{d, e\}}\left(a_{i}+b_{i}\right) t^{i}$, the set $R[t]$ of left polynomials in $t$ over $R$ is a ring. The evaluation $C(r)$ of $C$ at $r \in R$ is defined as

$$
C(r):=\sum_{i=0}^{d} c_{i} r^{i}
$$

Besides this "right evaluation" there is also a "left evaluation" $\sum_{i=0}^{d} r^{i} c_{i}$ which gives rise to a completely symmetric theory. A ring element $r$ is called a right zero of $C$ if $C(r)=0$ and a left zero if its left evaluation at $r$ vanishes. Since left evaluation and left zeros are not important for this paper, we introduce no special notation for them. We will often simple speak of "evaluation" and "zeros" instead of "right evaluation" and "right zeros".

Evaluation of $C$ at a fixed value $r \in R$ is not generally a ring homomorphism. For a counter example, take two non-commuting elements $r, q \in R$ and set $C:=C_{r} C_{q}$ where $C_{r}:=t-r$ and $C_{q}:=t-q$. We then have

$$
C(r)=r^{2}-(r+q) r+r q=r q-q r \neq 0 \quad \text { but } \quad C_{r}(r) C_{q}(r)=0
$$

because $C_{r}(r)=0$. However, we do have

$$
C(q)=q^{2}-(r+q) q+r q=0 .
$$

This is no coincidence but consequence of Theorem 2 below. Note that evaluation at $r$ is at least additive: For all $F, G \in R[t]$ we have $(F+G)(r)=F(r)+G(r)$.

A polynomial $F$ is called a right factor of $C$ if there exists a polynomial $Q$ such that $C=Q F$. Similarly, it is called a left factor if $C=F Q$. Polynomial division is possible in $R[t]$ but it is necessary to distinguish between a left and a right version and to take into account non-invertible coefficients.

Theorem 1. Given polynomials $F, G \in R[t]$ such that the leading coefficient of $G$ is invertible, there exist unique polynomials $Q_{\ell}, Q_{r}, S_{\ell}$, and $S_{r}$ such that $\operatorname{deg} S_{\ell}<\operatorname{deg} G, \operatorname{deg} S_{r}<\operatorname{deg} G$ and $F=Q_{\ell} G+S_{\ell}=$ $G Q_{r}+S_{r}$.

Definition 1. The polynomials $Q_{\ell}, Q_{r}$ in Theorem 1 are called left and right quotient, respectively. The polynomials $S_{\ell}$ and $S_{r}$ are called left and right remainder. We denote them by $Q_{\ell}=\operatorname{lquo}(F, G)$, $Q_{r}=\operatorname{rquo}(F, G), S_{\ell}=\operatorname{lrem}(F, G)$, and $S_{r}=\operatorname{rrem}(F, G)$, respectively.

Proof of Theorem 1. Standard proofs for existence also work in this case. We do not repeat them here but instead refer to Algorithm 1, the Euclidean Algorithm for left polynomial division. Its correctness is easy to see, the "right" version is explained in comments.

As to uniqueness, assume that there are two left quotients and remainders, that is, $F=Q_{1} G+S_{1}=$ $Q_{2} G+S_{2}$. This implies

$$
\left(Q_{1}-Q_{2}\right) G=S_{2}-S_{1} .
$$


Now if $Q_{1} \neq Q_{2}$, the polynomial on the left-hand side has degree greater than or equal to deg $G$ because the leading coefficient of $G$ is invertible. But the degree on the right-hand side is strictly smaller. Hence $Q_{1}=Q_{2}$ and also $S_{1}=S_{2}$. In the same way we can prove uniqueness of right quotient and remainder.

Remark 1. If the leading coefficient of $G$ fails to be invertible, neither existence nor uniqueness of quotient and remainder can be guaranteed. These phenomena will be illustrated in Examples 1 and 2 below (after suitable associative real algebras will be constructed).

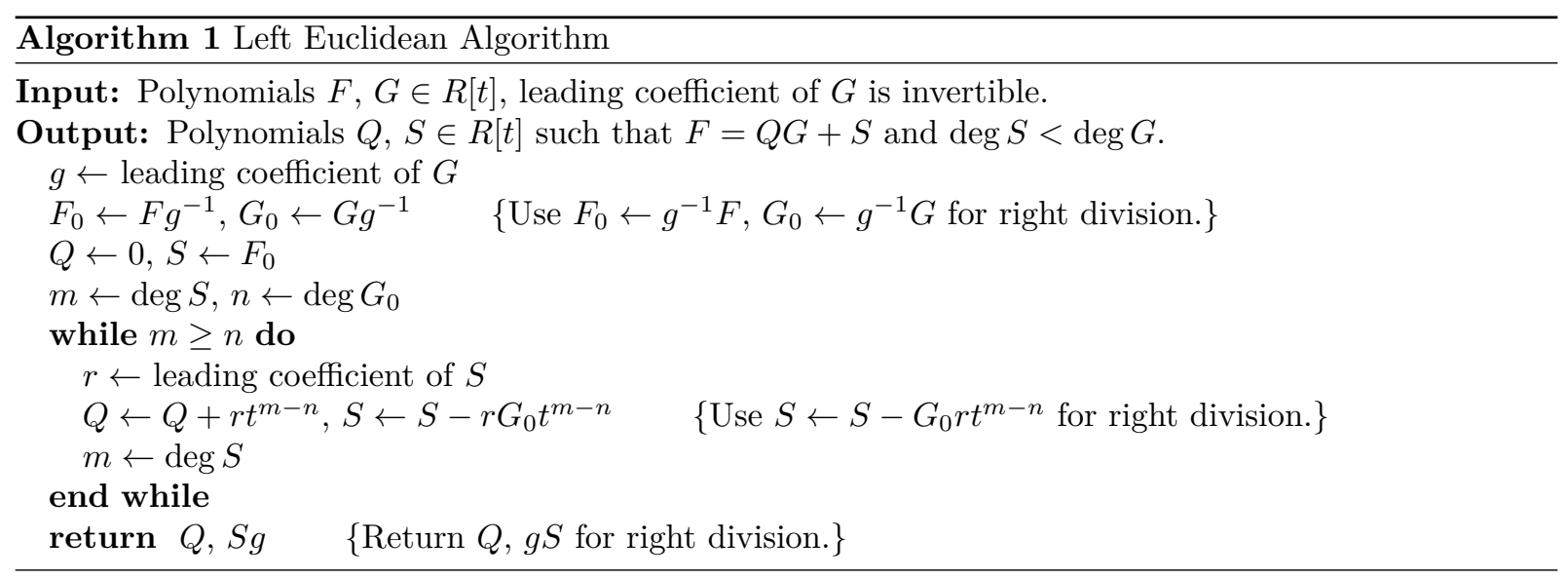

The next result has been shown in [2] for division rings but it holds true in more general rings (see [3] for the case of dual quaternions).

Theorem 2. The ring element $r \in R$ is a right zero of $C$ if and only if $t-r$ is a right factor of $C$.

Proof. Using polynomial division, we obtain $C=F+s$ where $F=Q(t-r)$ and $s \in R$. By uniqueness of polynomial division, $t-r$ is a right factor if and only if $s=0$. Writing $Q=\sum_{i=0}^{d} q_{i} t^{i}$, we compute

$$
Q(t-r)=\sum_{i=0}^{d}\left(q_{i} t^{i}\right)(t-r)=\sum_{i=0}^{d} q_{i} t^{i+1}-\sum_{i=0}^{d} q_{i} r t^{i}
$$

whence

$$
F(r)=\sum_{i=0}^{d} q_{i} r^{i+1}-\sum_{i=0}^{d} q_{i} r r^{i}=0 .
$$

From $C(r)=F(r)+s=s$ we infer that $r$ is a right zero of $C$ if and only $s=0$.

Theorem 2 has a corollary which is sometimes useful:

Corollary 1. If $F, G \in R[t]$ and $S=\operatorname{lrem}(F, G)$, then $F(r)=S(r)$ for every zero $r$ of $G$.

Proof. If $r$ is a zero of $G$, then $t-r$ is a right factor of $G$ and also of $Q G$ for $Q=\operatorname{lquo}(F, G)$. Hence, $F(h)=(Q G)(h)+S(h)=0+S(h)$.

Definition 2. We say that the polynomial $C \in R[t]$ of degree $n \geq 1$ admits a factorization if there exist ring elements $c_{n}, h_{1}, h_{2}, \ldots, h_{n}$ such that $C=c_{n}\left(t-h_{1}\right)\left(t-h_{2}\right) \cdots\left(t-h_{n}\right)$. 
It will simplify things a lot if the leading coefficient $c_{n}$ of $C$ is invertible. In this case, it is no loss of generality to assume $c_{n}=1$ because $C$ admits a factorization if and only if $c_{n}^{-1} C$ does. We will generally assume that $C$ is monic.

Theorem 2 relates zeros with linear right factors of $C$. Using Theorem 1 and Algorithm 1 it is possible to compute linear right factors from zeros. This situation is reminiscent of polynomial factorization over the complex numbers $\mathbb{C}$ but there are fundamental differences due to non-commutativity and existence of zero-divisors.

\section{Existence of Factorizations}

In the following, denote by $R$ a finite-dimensional associative real involutive algebra with multiplicative identity 1 and involution $\gamma$. The involution $\gamma: R \rightarrow R$ has the following properties:

- $\gamma \circ \gamma$ is the identity on $R$

- $\forall a, b \in R, \alpha, \beta \in \mathbb{R}: \gamma(\alpha a+\beta b)=\alpha \gamma(a)+\beta \gamma(b)$

- $\forall a, b \in R: \gamma(a b)=\gamma(b) \gamma(a)$

These properties already imply that $\gamma(1)=1$ :

$$
\forall a \in R: 1 \cdot \gamma(a)=\gamma(a) \Longrightarrow \gamma(1 \cdot \gamma(a))=\gamma(\gamma(a)) \Longrightarrow a \cdot \gamma(1)=a .
$$

We are going to prove existence results of factorizations of left polynomials over some $R$ for which the additional assumption $\gamma(a) a=a \gamma(a)$ holds for all $a \in R$. Theorem 3 below covers the case of division rings (real numbers, complex numbers and quaternions by Frobenius' Theorem) but in its formulation and proof we do not make direct use of properties of these number systems. The reason is that the corresponding Algorithm 2 may make sense in the presence of non-invertible elements as well. Variants and generalizations of Theorem 3 and Algorithm 2 with weaker assumptions are given in Section 6.

Since the center of the ring $R$ contains $\mathbb{R}$, any polynomial $C \in R[t]$ has a unique real monic factor of maximal degree. We denote this factor by $\operatorname{mrpf} C$ (the "maximal real polynomial factor"). For reasons of simplicity, we assume that it equals 1 .

Theorem 3. If a finite-dimensional associative real involutive algebra $R$ with involution $\gamma$ is

a) a division ring and

b) satisfies

$$
\forall a \in R: \nu(a):=\gamma(a) a=a \gamma(a) \in \mathbb{R},
$$

then every monic polynomial $C \in R[t]$ of positive degree and with $\operatorname{mrpf} C=1$ admits a factorization.

By Frobenius' Theorem (see for example [13]) $R$ is either the field of real or complex numbers or the skew field of quaternions. Hence, Theorem 3 does not present a new result. Moreover, the involution $\gamma$ is the usual complex or quaternion conjugation and requirement b) need not be stated as hypothesis. However, our formulation of Theorem 3 already takes into account later generalizations where condition a) will not be needed but condition b) will be crucial.

Let us drop for a moment the condition that $R$ is a division ring. If an involution $\gamma$ as in Theorem 3 is given, the inverse of $r \in R$ (if it exists) is $\gamma(r) / \nu(r)$. In particular, $r$ is invertible if and only if $\nu(r) \neq 0$ and $\gamma(r)$ is unique up to sign. If the involution $\gamma$ does not satisfy (1), we may instead consider the multiplicative semigroup

$$
R^{\gamma}:=\{a \in R \mid \gamma(a) a=a \gamma(a) \in \mathbb{R}\}
$$


Examples for semigroups of this type are the pin and spin groups of Clifford algebras. We may extend $\gamma$ to the involution

$$
R[t] \rightarrow R[t], \quad \sum_{i=0}^{n} c_{i} t^{i} \mapsto \sum_{i=0}^{n} \gamma\left(c_{i}\right) t^{i}
$$

for polynomials over $R$. By abuse of notation, we denote it by the same symbol. For $C \in R[t]$, condition (1) implies that the norm polynomial $\nu(C):=C \gamma(C)=\gamma(C) C$ of $C$ is in $\mathbb{R}[t]$. Also note that we may perform the semigroup construction of Equation (2) for polynomials:

$$
R^{\gamma}[t]:=\{C \in R[t] \mid \gamma(C) C=C \gamma(C) \in \mathbb{R}[t]\} .
$$

Remark 2. In this context, our general assumption that the polynomial to be factorized is monic, is an actual restriction. If $\nu(C) \neq 0$, there is a suitable fractional linear parameter transformation $t \mapsto(\alpha t+$ $\beta)(\gamma t+\delta)^{-1}$ with $\alpha, \beta, \gamma, \delta \in \mathbb{R}$ and $\alpha \delta-\beta \gamma \neq 0$ that makes the leading coefficient $g$ of $C^{\prime}:=(\gamma t+\delta)^{\operatorname{deg} C} C$ invertible. Any factorization of $g^{-1} C^{\prime}$ also gives rise to a factorization of $C$ and vice versa. If $\nu(C)=0$, no suitable fractional linear parametrization exists. Polynomials with the property $\nu(C)=0$ are not covered in this text. Their factorizability requires a separate investigation.

Lemma 1. Suppose that $R, C, \gamma$, and $\nu$ are as in Theorem 3 (but $R$ is not necessarily a division ring). If $M$ is a monic, quadratic and real factor of $\nu(C)$ and $S:=\operatorname{lrem}(C, M)$ satisfies $\nu(S) \neq 0$, then $S$ has a unique zero $h$ and $t-h$ is a right factor of $C$.

Proof. Using polynomial division we can find $Q, S \in R[t] \operatorname{such}$ that $C=Q M+S$ and $\operatorname{deg} S \leq 1$. Moreover, because of

$$
\nu(C)=(Q M+S) \gamma(Q M+S)=(Q M+S)(M \gamma(Q)+\gamma(S))=(\nu(Q) M+Q \gamma(S)+S \gamma(Q)) M+\nu(S),
$$

$M$ is also a factor of $\nu(S)$. Thus, there exists $c \in \mathbb{R}$ such that $\nu(S)=c M$. By assumption, $c \neq 0$ whence $S=s_{1} t+s_{0}$ with $s_{0}, s_{1} \in R$ and $\nu\left(s_{1}\right)=c \neq 0$. Hence, there is a unique zero $h=-s_{1}^{-1} s_{0}$ of $S$ and $t-h$ is not only a right factor of $S$ but also of $M$.

Proof of Theorem 3. We prove the theorem by induction on $n:=\operatorname{deg} C$. For $n=1$ the statement is obvious. For the induction step, we pick a quadratic factor $M$ of $\nu(C)$ and compute $S:=\operatorname{lrem}(C, M)$. The remainder polynomial always satisfies $\nu(S) \neq 0$ because $M$ cannot be a factor of $C$ and $R$ is assumed to be a division ring. Hence, we may use Lemma 1 to construct one right factor $t-h$. The induction hypothesis applied to lquo $(C, t-h)$ then guarantees existence of a factorization.

Our inductive proof of Theorem 3 gives rises to the recursive Algorithm 2 for computing factorizations of a polynomial $C \in R[t]$. It has been used in [3] to factor quaternion and certain dual quaternion polynomials. If $M \in \mathbb{R}[t]$ is of degree two, we denote the unique zero (according to Lemma 1 ) of $\operatorname{lrem}(C, M)$ by czero $(C, M)$. For two tuples $T_{1}$ and $T_{2}$ of polynomials we denote by $\left(T_{1}, T_{2}\right)$ their concatenation.

Remark 3. A few remarks on Algorithm 2 are in order:

- Because in each recursion, a quadratic factor $M$ of the norm polynomial $\nu(C)$ is chosen, the algorithm is not deterministic. In fact, it generically gives rise to a finite number of different factorizations. The total number of factorizations depends on the number of irreducible (over $\mathbb{R})$ real quadratic factors of $\nu(C)$, the number of real linear factors of $\nu(C)$ and their respective multiplicities.

- Algorithm 2 will produce all factorizations of $C$ : If $C=C^{\prime}(t-h)$, then $\nu(C)=\nu\left(C^{\prime}\right) \nu(t-h)$ and $\nu(t-h)$ is among the quadratic factors of $\nu(C)$. 


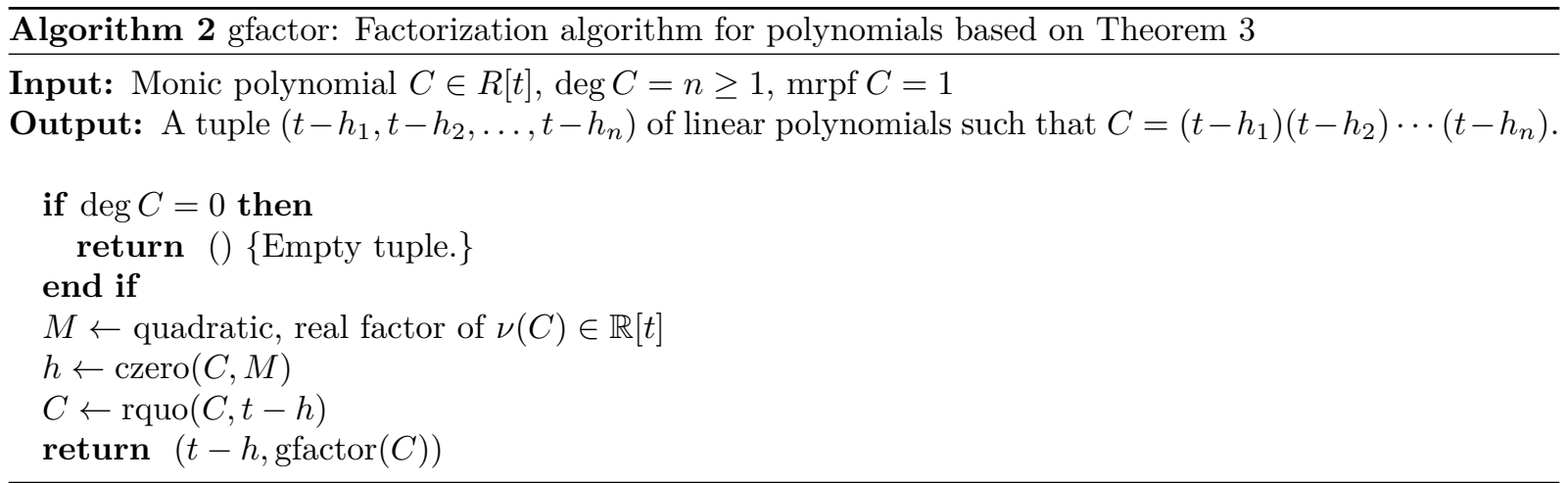

Also note that the assumption mrpf $C=1$ can be dropped for rings that contain the complex numbers $\mathbb{C}$ as a subring. We may combine any factorization $\operatorname{mrpf} C=\left(t-z_{1}\right)\left(t-z_{2}\right) \cdots\left(t-z_{\ell}\right)$ over (the subring isomorphic to $) \mathbb{C}$ with any factorization lquo $(C, \operatorname{mrpf} C)=\left(t-h_{1}\right)\left(t-h_{2}\right) \cdots\left(t-h_{m}\right)$ to obtain the factorization $C=\left(t-z_{1}\right)\left(t-z_{2}\right) \cdots\left(t-z_{\ell}\right)\left(t-h_{1}\right)\left(t-h_{2}\right) \cdots\left(t-h_{m}\right)$.

Algorithm 2 is based on a factorization of the real polynomial $\nu(C)$ over $\mathbb{R}$. For moderate polynomial degrees, numeric factorization of real polynomials is always possible $([14,15])$, but the ensuing polynomial division may be tricky. Without going into detail, we mention that it is possible to make Algorithm 2 numerically stable by using the evaluation-interpolation univariate polynomial division algorithm (e.g., the fast and robust algorithm based on the Fast Fourier Transform in $[16,17,18]$ ) to compute $h \in R$ such that $M$ divides $C(t-\gamma(h))$. Because of $M=(t-h)(t-\gamma(h))$, this is equivalent to the computation in Algorithm 2.

\section{Factorization Examples}

In this section, we explicitly construct some rings over Clifford algebras and present factorization examples for polynomials over those rings. Note that not all polynomials in these examples satisfy the requirements of Theorem 3 and Algorithm 2. Nonetheless, it might be possible to use Algorithm 2 to compute factorizations.

4.1. Clifford Algebras. Our brief introduction to Clifford algebras follows [19] and [20, Section 9.1]. In the real vector space $\mathbb{R}^{n}$ we consider a quadratic form $\varrho: \mathbb{R}^{n} \rightarrow \mathbb{R}$. With respect to a basis $\left(e_{1}, e_{2}, \ldots, e_{n}\right)$ it is described by a matrix $Q \in \mathbb{R}^{n \times n}$ via $\varrho\left(e_{i}, e_{j}\right)=e_{i}^{\top} \cdot Q \cdot e_{j}$. The defining relations for the Clifford algebra are

$$
e_{i} e_{j}+e_{j} e_{i}:=2 e_{i}^{\top} \cdot Q \cdot e_{j} \text { for all } i, j \in\{1,2, \ldots, n\} .
$$

With respect to a different basis, the same quadratic form is described by a congruent matrix. Hence, by Sylvester's Theorem of Inertia, there is a basis such that $Q$ is diagonal with the first $p$ diagonal entries equal to 1 , the next $q$ diagonal entries equal to -1 and the remaining $r=n-p-q$ diagonal entries equal to 0 . We assume that this is the case for the chosen basis $\left(e_{1}, e_{2}, \ldots, e_{n}\right)$, which, together with (4), implies $e_{i} e_{j}=-e_{j} e_{i}$ whenever $i \neq j$. For the product of successive basis elements we also use the shorthand notation

$$
e_{12 \ldots k}:=e_{1} e_{2} \cdots e_{k} \quad \text { for } 1 \leq k \leq n
$$


The span of all these element with multiplicative structure given by (4) is called a Clifford algebra and will be denoted by $C \ell_{(p, q, r)}$. An element of $C \ell_{(p, q, r)}$ can be written as

$$
r=a_{0}+\sum_{k=1}^{n} \sum_{i_{1}<i_{2}<\cdots<i_{k}} a_{i_{1} i_{2} \ldots i_{k}} e_{i_{1} i_{2} \ldots i_{k}}
$$

where $a_{0}, a_{1}, \ldots, a_{12 \ldots n} \in \mathbb{R}$ and all summation indices are between 1 and $n$. Often, the real unit 1 is identified with an additional basis element $e_{0}$ whence above sum starts with $r=a_{0} e_{0}+\ldots$ We will usually follow this convention.

The conjugation $r \mapsto r^{*}$ in $C \ell_{(p, q, r)}$ is the $\mathbb{R}$-linear anti-automorphism defined by

$$
\left(e_{i_{1}} e_{i_{2}} \cdots e_{i_{k}}\right)^{*}:=(-1)^{k}\left(e_{i_{k}} \cdots e_{i_{2}} e_{i_{1}}\right) .
$$

It gives rise to the norm $N(r):=r r^{*}$. Elements in the span of $e_{1}, e_{2}, \ldots, e_{n}$ are called vectors and we identify them with elements of $\mathbb{R}^{n}$. The even sub-algebra $C \ell_{(p, q, r)}^{+}$of $C \ell_{(p, q, r)}$ is the sub-algebra generated by basis elements $e_{i_{1} i_{2} \ldots i_{k}}$ with $k$ even (and by $e_{0}$ ). The spin group is

$$
\operatorname{Spin}_{(p, q, r)}:=\left\{r \in C \ell_{(p, q, r)}^{+} \mid N(r)= \pm 1, \forall v \in \mathbb{R}^{n}: r v r^{*} \in \mathbb{R}^{n}\right\} .
$$

The map $\sigma_{r}: v \mapsto r v r^{*}$ is called the sandwich operator.

Clifford algebras comprise several well-known algebraic structures. In the context of polynomial factorization, algebras that permit the construction of isomorphisms to transformation groups of Euclidean and non-Euclidean spaces are of special interest. There, factorization corresponds to the decomposition of rational motions into products of elementary motions.

Quaternions. An element of $C \ell_{(0,3,0)}^{+}$can be written as $r=a_{0} e_{0}+a_{1} e_{12}+a_{2} e_{13}+a_{3} e_{23}$. We have $e_{12}^{2}=e_{12} e_{12}=-e_{12} e_{21}=-1$ and also $e_{13}^{2}=e_{23}^{2}=-1$. This even Clifford sub-algebra is isomorphic to the quaternion algebra $\mathbb{H}$. The basis elements $e_{12}, e_{13}$, and $e_{23}$ correspond, in that order, to the quaternion units $\mathbf{i}, \mathbf{j}$, and $\mathbf{k}$, respectively. We will usually use the quaternion notation and write $r=a_{0}+a_{1} \mathbf{i}+a_{2} \mathbf{j}+a_{3} \mathbf{k}$. For $r$ as above, $N(r)=a_{0}^{2}+a_{1}^{2}+a_{2}^{2}+a_{3}^{2} \geq 0$ and $\sigma_{r}(v) \in \mathbb{R}^{3}$ for all $v \in \mathbb{R}^{3}$. Hence, the only defining condition for spin group elements is $N(r)=1$. The map $r \rightarrow \sigma_{r}$ is an isomorphism between $\operatorname{Spin}_{(0,3,0)} /\{ \pm 1\}$ and $\mathrm{SO}(3)$ and accounts for the importance of $C \ell_{(0,3,0)}$ in spatial kinematics.

Also note that the factor group $\mathbb{H}^{\times} / \mathbb{R}^{\times}$of the multiplicative quaternion group modulo the multiplicative reals is isomorphic to $\mathrm{SO}(3)$ via the map that sends $r \in \mathbb{H}^{\times}$to the map $x \in \mathbb{R}^{3} \mapsto \sigma_{r}(x) / N(r)$. This isomorphism is more useful in the context of quaternion polynomial factorization $\left(C C^{*}=1\right.$ is only satisfied by the constant polynomials $C= \pm 1$ ).

Split Quaternions. Also kinematics in planar hyperbolic geometry may be treated by means of a Clifford algebra. The construction is similar to the construction of $\mathbb{H}$ but is based on the even Clifford algebra $C \ell_{(1,2,0)}^{+}$. We set $\mathbf{i}_{s}:=e_{12}, \mathbf{j}_{s}:=e_{13}, \mathbf{k}_{s}:=e_{23}$ and denote the algebra generated by $1, \mathbf{i}_{s}, \mathbf{j}_{s}$ and $\mathbf{k}_{s}$ by $\mathbb{S}_{\text {. }}$ The norm of $r=a_{0}+a_{1} \mathbf{i}_{s}+a_{2} \mathbf{j}_{s}+a_{3} \mathbf{k}_{s} \in \mathbb{S}$ equals

$$
N(r)=\left(a_{0}+a_{1} \mathbf{i}_{s}+a_{2} \mathbf{j}_{s}+a_{3} \mathbf{k}_{s}\right)\left(a_{0}-a_{1} \mathbf{i}_{s}-a_{2} \mathbf{j}_{s}-a_{3} \mathbf{k}_{s}\right)=a_{0}^{2}-a_{1}^{2}-a_{2}^{2}+a_{3}^{2} .
$$

We see that $N\left(\sigma_{r}(v)\right)=N(r)^{2} N(v)$ equals $N(v)$ for all vectors $v \in \mathbb{R}^{3}$ if and only if $N(r)= \pm 1$. Hence $\operatorname{Spin}_{(1,2,0)}$ is isomorphic to a transformation subgroup of planar hyperbolic geometry. In contrast to the quaternions $\mathbb{H}$, the norm of these so-called split quaternions can attain negative values. As in the case of quaternions we have $r^{-1}=r^{*} / N(r)$ but the inverse element exists only if $N(r) \neq 0$. In particular, $\mathbb{S}$ is not a division ring and Theorem 3 is not generally applicable. 
Dual Quaternions. An isomorphism from a Clifford algebra based group to the group SE(3) of rigid body displacements requires a more elaborate construction. An element of $C \ell_{(3,0,1)}^{+}$is of the shape

$$
r=a_{0} e_{0}+a_{3} e_{12}-a_{2} e_{13}+b_{1} e_{14}+a_{1} e_{23}+b_{2} e_{24}+b_{3} e_{34}-b_{0} e_{1234}
$$

with $a_{0}, a_{1}, a_{2}, a_{3}, b_{0}, b_{1}, b_{2}, b_{3}$ in $\mathbb{R}$. Its norm equals

$$
N(r)=\left(a_{0}^{2}+a_{1}^{2}+a_{2}^{2}+a_{3}^{2}\right) e_{0}-\left(a_{0} b_{0}+a_{1} b_{1}+a_{2} b_{2}+a_{3} b_{3}\right) e_{1234}
$$

The spin group conditions are

$$
a_{0}^{2}+a_{1}^{2}+a_{2}^{2}+a_{3}^{2}=1, \quad a_{0} b_{0}+a_{1} b_{1}+a_{2} b_{2}+a_{3} b_{3}=0
$$

and the restriction of the conjugation map $r \mapsto r^{*}$ to $\operatorname{Spin}_{(3,0,1)}$ (but not its extension to $\mathbb{D H H}$ ) qualifies to play the role of $\gamma$ in Theorem 3 .

The algebra of dual quaternions $\mathbb{D H}$ is obtained from $\mathbb{H}$ by extension of scalars from the real numbers to the dual numbers $\mathbb{D}=\mathbb{R}[\varepsilon] /\left\langle\varepsilon^{2}\right\rangle$. By Equation (3.3) of [19], the map

$$
\begin{aligned}
a_{0} e_{0}+a_{3} e_{12}-a_{2} e_{13}+b_{1} e_{14}+a_{1} e_{23}+b_{2} e_{24}+b_{3} e_{34} & -b_{0} e_{1234} \\
& \mapsto a_{0}+a_{1} \mathbf{i}+a_{2} \mathbf{j}+a_{3} \mathbf{k}+\varepsilon\left(b_{0}+b_{1} \mathbf{i}+b_{2} \mathbf{j}+b_{3} \mathbf{k}\right)
\end{aligned}
$$

is an isomorphism between $C \ell_{(3,0,1)}^{+}$and the algebra $\mathbb{D H}$ of dual quaternions. Again, we will prefer the dual quaternion notation in this text. The spin group $\operatorname{Spin}_{(3,0,1)}$ is isomorphic to $\operatorname{SE}(3)$ by virtue of the action $\left(x_{1}, x_{2}, x_{3}\right) \mapsto\left(y_{1}, y_{2}, y_{3}\right)$ where

$$
1+\varepsilon\left(y_{1} \mathbf{i}+y_{2} \mathbf{j}+y_{3} \mathbf{k}\right)=(a-\varepsilon b)\left(1+\varepsilon\left(x_{1} \mathbf{i}+x_{2} \mathbf{j}+x_{3} \mathbf{k}\right)\right)\left(a^{*}+\varepsilon b^{*}\right)
$$

and $r=a+\varepsilon b \in \operatorname{Spin}_{(3,0,1)}$. This is not quite the sandwich operator but reduces to $\sigma_{a}$ for pure quaternions $(b=0)$. The translation vector equals $a b^{*}-b a^{*}$. More generally, transformation groups of arbitrary Euclidean spaces can be modeled by spin groups of Clifford algebras [19, Chapter 3].

Now that we have explicitly constructed several associative real algebras, we are able to illustrate Remark 1 on non-existence or non-uniqueness of quotient and remainder by concrete examples:

Example 1. Division of $F=t \in \mathbb{D} \mathbb{H}[t]$ by $G=t \varepsilon \in \mathbb{D} \mathbb{H}[t]$ is not possible; quotient and remainder do not exist.

Example 2. With

$$
\begin{array}{ll}
Q_{1}=t+1+3 \mathbf{i}_{s}+\mathbf{j}_{s}+2 \mathbf{k}_{s}, & S_{1}=1+\mathbf{j}_{s}, \\
Q_{2}=t+5-\mathbf{i}_{s}+\mathbf{j}_{s}+2 \mathbf{k}_{s}, & S_{2}=1-3 \mathbf{j}_{s}-4 \mathbf{k}_{s},
\end{array}
$$

and $G=\left(1+\mathbf{i}_{s}\right) t+2 \mathbf{j}_{s}-\mathbf{k}_{s}$ we have

$$
F:=Q_{1} G+S_{1}=Q_{2} G+S_{2}=\left(1+\mathbf{i}_{s}\right) t^{2}+\left(4+4 \mathbf{i}_{s}+5 \mathbf{j}_{s}+2 \mathbf{k}_{s}\right) t+5-5 \mathbf{i}_{s}+6 \mathbf{j}_{s}-7 \mathbf{k}_{s} .
$$

Neither quotient nor remainder of the division of $F$ by $G$ are unique.

4.2. Factorization examples. We now illustrate some peculiarities of polynomial factorization over Clifford algebras. We consider left polynomials over quaternions, split quaternions and dual quaternions and demonstrate examples of typical and special factorizations. Verifying correctness of the presented factorizations is straightforward. Often, Algorithm 2 could be used for computing factorizations, even if not all requirements were fulfilled.

Example 3. The polynomial $C=t^{2}-(2 \mathbf{i}+\mathbf{j}+2) t+2 \mathbf{i}+\mathbf{j}+2 \mathbf{k}+1 \in \mathbb{H}[t]$ admits the two factorizations

$$
C=(t-2 \mathbf{i}-1)(t-\mathbf{j}-1)=\left(t-\frac{4}{5} \mathbf{i}+\frac{3}{5} \mathbf{j}-1\right)\left(t-\frac{6}{5} \mathbf{i}-\frac{8}{5} \mathbf{j}-1\right) .
$$

Other factorizations do not exist. This is a generic case, factorizations can be computed by Algorithm 2 . 
Example 4. The polynomial $C=t^{3}-t^{2}+t-1 \in \mathbb{R}[t]$ admits the factorizations

$$
C=(t-1)(t-h)\left(t-h^{*}\right)
$$

where $h \in \mathbb{U}$ and

$$
\mathbb{U}:=\left\{h \in \mathbb{H} \mid h^{2}=-1\right\}=\left\{h_{1} \mathbf{i}+h_{2} \mathbf{j}+h_{3} \mathbf{k} \mid h_{1}^{2}+h_{2}^{2}+h_{3}^{2}=1\right\} .
$$

All other factorizations are obtained by suitable permutations of the three factors in (5). These factors were found by factorizing $C$ over $\mathbb{C}$ as $C=(t-1)(t-\mathrm{i})(t+\mathrm{i})$ and replacing the complex unit i with $h$. Correctness of this construction follows from $h^{2}=\mathrm{i}^{2}=-1$. As far as factorization of real polynomials is concerned, there is no essential algebraic difference between $h \in \mathbb{U}$ and i.

The factorization theory of general quaternion polynomials is well understood (see [1]). Given $C \in \mathbb{H}[t]$, write $C=F G$ with $F=\operatorname{mrpf} C$. If $F=\prod_{\ell}\left(t-t_{\ell}\right) \prod_{m}\left(t-z_{m}\right)\left(t-\bar{z}_{m}\right)$ with $t_{\ell} \in \mathbb{R}$ and $z_{m}=x_{m}+\mathrm{i} y_{m} \in \mathbb{C}$ is the factorization of $F$ over $\mathbb{C}$, all factorizations over $\mathbb{H}$ are obtained by replacing $z_{m}=x_{m}+\mathrm{i} y_{m}$ with $x_{m}+h_{m} y_{m}$ and $h_{m} \in \mathbb{U}$. All factorizations of $G$ are obtained by Algorithm 2 with different choices of the quadratic factor $M$ at each recursion level. Depending on the number of different quadratic factors (multiplicities of these factors), there exist between 1 and $(\operatorname{deg} G)$ ! different factorizations of $G$. All factorizations of $C=F G$ are obtained by combining factorizations of $F$ with factorizations of $G$ in an obvious way.

Example 5. The polynomial $C=t^{2}-\left(2+2 \mathbf{i}_{s}+\mathbf{j}_{s}\right) t+2 \mathbf{i}_{s}+\mathbf{j}_{s}+2 \mathbf{k}_{s}+1 \in \mathbb{S}$ admits precisely six different factorizations:

$$
\begin{aligned}
C & =\left(t-\mathbf{j}_{s}-1\right)\left(t-2 \mathbf{i}_{s}-1\right), \\
& =\left(t-\frac{6}{5} \mathbf{i}_{s}-\frac{8}{5} \mathbf{j}_{s}-1\right)\left(t-\frac{4}{5} \mathbf{i}_{s}+\frac{3}{5} \mathbf{j}_{s}-1\right), \\
& =\left(t-\frac{3}{2} \mathbf{i}_{s}+\frac{1}{2} \mathbf{j}_{s}-\frac{3}{2} \mathbf{k}_{s}+\frac{1}{2}\right)\left(t-\frac{1}{2} \mathbf{i}_{s}-\frac{3}{2} \mathbf{j}_{s}+\frac{3}{2} \mathbf{k}_{s}-\frac{5}{2}\right), \\
& =\left(t-\frac{3}{2} \mathbf{i}_{s}+\frac{1}{2} \mathbf{j}_{s}+\frac{3}{2} \mathbf{k}_{s}-\frac{5}{2}\right)\left(t-\frac{1}{2} \mathbf{i}_{s}-\frac{3}{2} \mathbf{j}_{s}-\frac{3}{2} \mathbf{k}_{s}+\frac{1}{2}\right), \\
& =\left(t-\frac{1}{2} \mathbf{i}_{s}-\frac{3}{2} \mathbf{j}_{s}+\frac{1}{2} \mathbf{k}_{s}-\frac{1}{2}\right)\left(t-\frac{3}{2} \mathbf{i}_{s}+\frac{1}{2} \mathbf{j}_{s}-\frac{1}{2} \mathbf{k}_{s}-\frac{3}{2}\right), \\
& =\left(t-\frac{1}{2} \mathbf{i}_{s}-\frac{3}{2} \mathbf{j}_{s}-\frac{1}{2} \mathbf{k}_{s}-\frac{3}{2}\right)\left(t-\frac{3}{2} \mathbf{i}_{s}+\frac{1}{2} \mathbf{j}_{s}+\frac{1}{2} \mathbf{k}_{s}-\frac{1}{2}\right) .
\end{aligned}
$$

In spite of $\mathbb{S}$ failing to be a division ring, above factorizations can be computed by means of Algorithm 2 . The number of six factorizations is related to the fact that $\nu(C)$ is the product of four linear polynomials $t, t+1, t-2$, and $t-3$. Hence, there exist six pairs $\left(M_{1}, M_{2}\right)$ of quadratic factors such that $\nu(C)=M_{1} M_{2}$ :

$$
\begin{aligned}
&\left(M_{1}, M_{2}\right) \in\{(t(t+1),(t-2)(t-3)),(t(t-2),(t+1)(t-3)),(t(t-3),(t+1)(t-2)), \\
&((t+1)(t-2), t(t-3)),((t+1)(t-3), t(t-2)),((t-2)(t-3), t(t+1))\} .
\end{aligned}
$$

The sub-algebra $\left\langle 1, \mathbf{k}_{s}\right\rangle$ is isomorphic to $\mathbb{C}$. Therefore, a real polynomial can be factored over $\mathbb{S}$ by replacing the complex unit i with $\mathbf{k}_{s}$. However, not all monic polynomials in $\mathbb{S}[t]$ admit factorizations, as the next example shows.

Example 6. The polynomial $C=t^{2}+2 \mathbf{i}_{s}$ does not admit a factorization. This can be proved by means of Theorem 2. Comparing coefficients on both sides of $C\left(x_{0}+x_{1} \mathbf{i}_{s}+x_{2} \mathbf{j}_{s}+x_{3} \mathbf{k}_{s}\right)=0$ we arrive at a system of algebraic equations in $x_{0}, x_{1}, x_{2}, x_{3}$ that has no real solutions. On the other hand, Algorithm 2 gives $t^{2}+2 \mathbf{k}_{s}=\left(t-\mathbf{k}_{s}+1\right)\left(t+\mathbf{k}_{s}-1\right)=\left(t+\mathbf{k}_{s}-1\right)\left(t-\mathbf{k}_{s}+1\right)$.

As for polynomials in $\mathbb{D} \mathbb{H}[t]$, even stranger examples exist:

Example 7. The polynomial $C=t^{2}+\varepsilon \in \mathbb{D} \mathbb{H}[t]$ admits no factorization. This can be shown in a similar way as in Example 6. 
Example 8. The polynomial $C=t^{2}+1-\varepsilon(\mathbf{j} t-\mathbf{i}) \in \mathbb{D} \mathbb{H}$ has the infinitely many factorizations

$$
C=(t-\mathbf{k}+\varepsilon(a \mathbf{i}+(b-1) \mathbf{j}))(t+\mathbf{k}-\varepsilon(a \mathbf{i}+b \mathbf{j})) \quad \text { where } \quad a, b \in \mathbb{R} .
$$

Example 9. The polynomial $C=t^{2}+1+\varepsilon \mathbf{i}$ lies in the subset $\{C \in \mathbb{D} \mathbb{H}[t] \mid \nu(C) \in \mathbb{R}[t]\}$ of real norm polynomials but admits no factorization into linear factors belonging to this subset (compare also with Example 12). Nonetheless, it admits two two-parametric families of factorizations over $\mathbb{D H}$ :

$$
\begin{aligned}
C & =\left(t+\mathbf{i}+\varepsilon\left(a \mathbf{j}+b \mathbf{k}-\frac{1}{2}\right)\right)\left(t-\mathbf{i}-\varepsilon\left(a \mathbf{j}+b \mathbf{k}-\frac{1}{2}\right)\right) \\
& =\left(t-\mathbf{i}+\varepsilon\left(a \mathbf{j}+b \mathbf{k}+\frac{1}{2}\right)\right)\left(t+\mathbf{i}-\varepsilon\left(a \mathbf{j}+b \mathbf{k}+\frac{1}{2}\right)\right)
\end{aligned}
$$

with arbitrary $a, b \in \mathbb{R}$.

\section{Application in Mechanism Science}

Factorization in (certain subsets of) Clifford algebras that are isomorphic to transformation groups has important applications in kinematics and mechanism science. The polynomial $C$ parameterizes a rational motion (all point trajectories are rational curves), the factorization corresponds to the decomposition of this motion into the product of "elementary motions" which are parameterized by the linear factors of the form $t-h$.

In $\mathbb{H}, \mathbb{S}$, and $\mathbb{D} \mathbb{H}$ two elements $h$ and $h^{*}$ commute whence

$$
(t-h)\left(h-h^{*}\right)\left(t-h^{*}\right)=\left(h-h^{*}\right)\left(t^{2}-\left(h+h^{*}\right) t+h h^{*}\right)=\left(h-h^{*}\right) \nu(t-h) .
$$

This shows that $c:=h-h^{*}$ and $\sigma_{t-h}(c)$ are equal up to multiplication with $\nu(t-h)$. This polynomial is real for quaternions and split quaternions. For dual quaternions we add $\nu(t-h) \in \mathbb{R}[t]$ as an assumption. Then $c$ is fixed under the spin group action of $t-h$ for any $t \in \mathbb{R}$. In case of $\mathbb{H}$ or $\mathbb{S}, c$ is a fix point of all displacements $t-h, t \in \mathbb{R}$. Generically, it is the only fix point in $\mathbb{H}$ and one or one of three fix points in $\mathbb{S}$. From this, we may already infer that $t-h$ describes a rotation in spherical space or in the hyperbolic plane. In $\mathbb{D H}$, the interpretation is similar but Equation (7) describes the action of the displacement $t-h$ on the line with Plücker coordinate vector c. (More precisely, if $c=a+\varepsilon b$, the line's Plücker coordinate vector according to the convention of $[21]$ is $[a,-b]$.) The straight line $c$ remains fixed and it is the axis of all spatial rotations described by $t-h$ for $t$ varying in $\mathbb{R}$.

Hence, factorization of a polynomial $C$ in $\mathbb{H}$, $\mathbb{S}$, or $\mathbb{D} \mathbb{H}$ (with the additional constraint $\nu(C) \in \mathbb{R}[t]$ ) corresponds to the decomposition of the motion parameterized by $C$ into a sequence of coupled rotations (translations in exceptional cases). Let us illustrate this with an example from mechanism science.

The sub-algebra $\langle 1, \mathbf{i}, \varepsilon \mathbf{j}, \varepsilon \mathbf{k}\rangle$ of $\mathbb{D} \mathbb{H}$ modulo the real multiplicative group $\mathbb{R}^{\times}$is isomorphic to $\operatorname{SE}(2)$. A generic quadratic polynomial $C$ in this sub-algebra admits two factorizations

$$
C=\left(t-h_{1}\right)\left(t-h_{2}\right)=\left(t-k_{1}\right)\left(t-k_{2}\right)
$$

(see Corollary 2 below). Each factorization corresponds to the composition of two rotations and both compositions result in the same motion. Hence, we may rigidly connect the centers of $h_{1}, h_{2}, k_{2}$ and $k_{1}$ (in that order) to obtain a four-bar linkage. Its middle link performs the motion parameterized by $C$. This is illustrated in Figure 1, left. It can be shown that the four-bar linkage is an anti-parallelogram [7]. A similar decomposition is not possible for the polynomial of Example 9.

The same construction is possible in $\mathbb{H}$ and $\mathbb{S}$ to obtain spherical and hyperbolic anti-parallelogram linkages (four-bar linkages with equal opposite sides) in the respective geometry. In case of $\mathbb{S}$, it is necessary to use the more general "universal hyperbolic geometry" in the sense of [22] in order to avoid awkward in-equality constraints. Figure 1, right, displays an example in the Cayley-Klein model of hyperbolic geometry with absolute circle (or null circle) $N$. Note that this example admits precisely two factorizations and gives rise to a unique four-bar linkage. The six factorizations of the polynomial of 


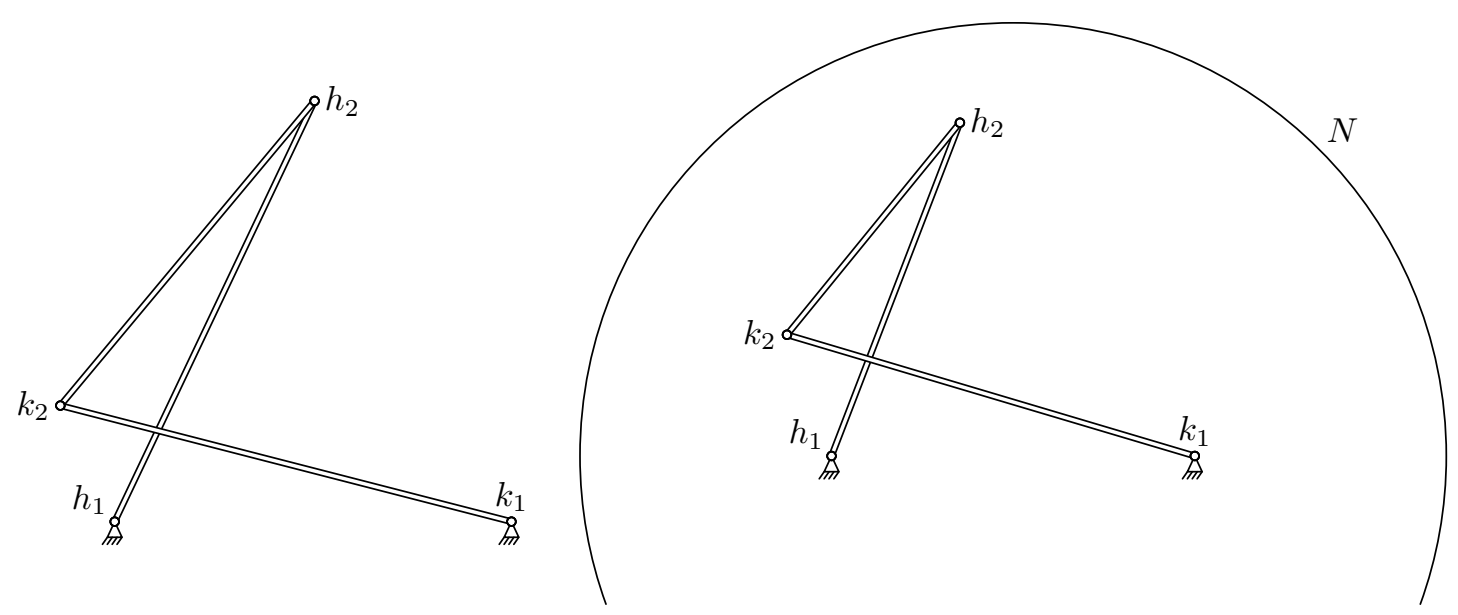

FiguRE 1. Anti-parallelogram mechanism in Euclidean geometry (left) and hyperbolic geometry (right)

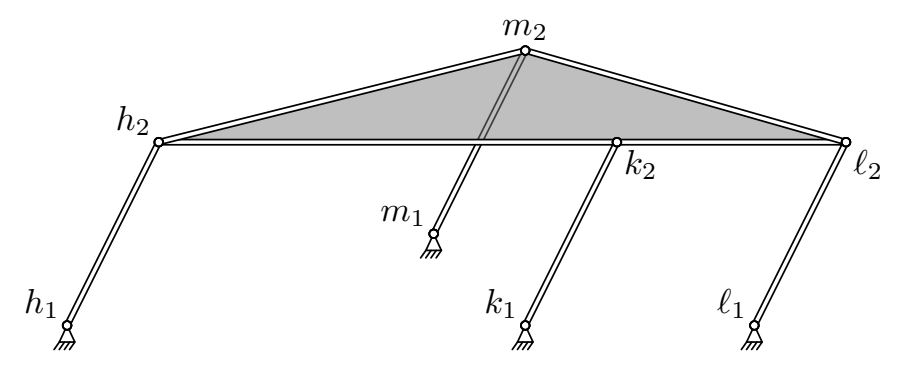

Figure 2. Parallelogram linkage

Example 5 give rise to a "four-bar linkage" with six possible legs. It cannot be visualized in traditional hyperbolic geometry because all rotation centers lie in the exterior of $N$ but is perfectly valid in universal hyperbolic geometry. A more detailed investigation of the underlying geometry of these factorizations is given in [23].

The polynomial of Example 8 parameterizes a circular translation. This motion can be generated by a parallelogram linkage (Figure 2) which, indeed, admits infinitely many legs, each corresponding to one of the infinitely many factorizations

$$
C=\left(t-h_{1}\right)\left(t-h_{2}\right)=\left(t-k_{1}\right)\left(t-k_{2}\right)=\left(t-\ell_{1}\right)\left(t-\ell_{2}\right)=\left(t-m_{1}\right)\left(t-m_{2}\right) \ldots
$$

The relevance of polynomial factorization in mechanism science goes beyond above simple examples (see for example $[24,5,6,11]$ ). It provides a more or less automatic way to construct linkages from rational motions. One example related to a rational version of Kempe's Universality Theorem is depicted in Figure 3. Any rational planar or spatial curve (an ellipse in Figure 3) can be drawn by a scissor like linkage whose number of joints is linear in the curve degree $[7,8]$. 

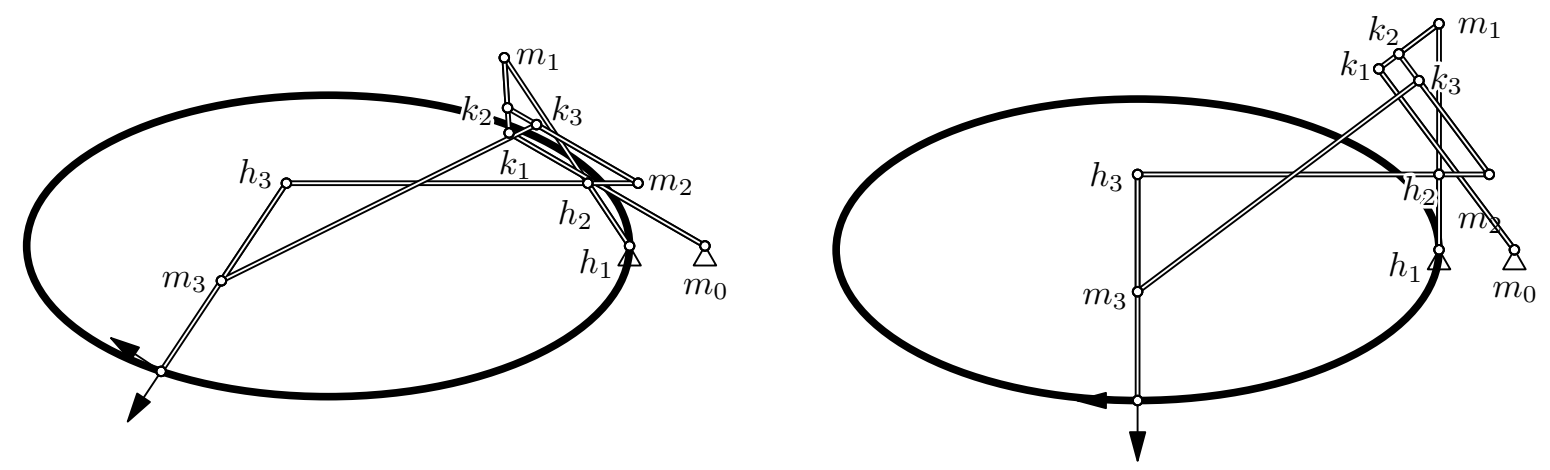

Figure 3. Scissor linkage to draw an ellipse.

\section{More Factorization Results and Examples}

It is unsatisfactory that Theorem 3 and Algorithm 2 are limited to division rings only. A detailed inspection of the proof of Theorem 3 shows that the property of $R$ being a division ring guarantees existence of an unique zero of the remainder polynomial $S$. However, as already demonstrated, Algorithm 2 may work in more general circumstances and even if it fails, factorizations may exists. In this section, we present miscellaneous existence and non-existence results for factorizations of polynomials in a certain subset of a finite-dimensional associative real involutive algebra whose involution $\gamma$ does not generally satisfy Equation (1), but $\gamma$ restricted to this subset does.

Note that Clifford algebras allow simple constructions of subsets that fall into this category. We define a suitable involution $\gamma$ by defining $\gamma\left(e_{\ell}\right)=e_{\ell}$ or $\gamma\left(e_{\ell}\right)=-e_{\ell}$ for $\ell \in\{0,1, \ldots, n\}$ and then extended $\gamma$ to the complete Clifford algebra by $\mathbb{R}$-linearity and the property $\gamma(a b)=\gamma(b) \gamma(a)$. The subset $R^{\gamma}$ defined in Equation (2) then satisfies all general assumptions of this section.

6.1. Applicability of Algorithm 2. An obvious pre-requisite for Algorithm 2 is that $\nu(C)$ is a nonzero real polynomial. The non-vanishing of $\nu(C)$ is guaranteed by our restriction to monic polynomials (compare Remark 2). The crucial property that then ensures applicability of (one iteration of) Algorithm 2 is that $S=\operatorname{lrem}(C, M)$ has a unique zero $(\operatorname{czero}(C, M)$ is well-defined). If this is the case, Algorithm 2 produces polynomials $C^{\prime}, t-h$ which are again in $R^{\gamma}[t]$ (even if $S$ is not): By construction $(t-h) \gamma(t-h)=$ $M \in \mathbb{R}[t]$ whence $\nu\left(C^{\prime}\right)$ must be real as well. In particular, $C^{\prime}$ is suitable as input for a further iteration of Algorithm 2. In order to have a convenient notion for the vanishing of the remainder polynomial, we state the following definition.

Definition 3. Given two polynomials $F, G \in R[t]$ where the leading coefficient of $G$ is invertible, $G$ is called a left pseudofactor of $F$, if $\operatorname{rrem}(F, G)$ has vanishing norm and a right pseudofactor of $F$, if $\operatorname{lrem}(F, G)$ has vanishing norm.

Obviously, left and right factors are also left and right pseudofactors, respectively. If a left pseudofactor is real then it is also a right pseudofactor and vice versa. In this case we simply speak of a pseudofactor. With the help of the involution $\gamma$, real pseudofactors can be found by factorizing $\nu(C)$ :

Theorem 4. A real pseudofactor of $C$ is a factor of $\nu(C)$. 
Proof. If $M$ is a real pseudofactor of $C$, there exist $Q, S \in R[t]$ with $C=Q M+S$, $\operatorname{deg} S<\operatorname{deg} M$, and $\nu(S)=0$. But then

$$
\begin{aligned}
& \nu(C)=C \gamma(C)=(Q M+S) \gamma(Q M+S) \\
& \quad=\nu(Q) M^{2}+(Q \gamma(S)+S \gamma(Q)) M+\underbrace{\nu(S)}_{=0}=(\nu(Q) M+Q \gamma(S)+S \gamma(Q)) M
\end{aligned}
$$

and $M$ is indeed a factor of $\nu(C)$.

As shown in [3], Algorithm 2 works for polynomials in an important subsemigroup of $\mathbb{D} \mathbb{H}[t]$ that have no real pseudofactors.

Definition 4. A polynomial $C=P+\varepsilon Q \in \mathbb{D} \mathbb{H}[t]$ with $P, Q \in \mathbb{H}[t]$ is called a motion polynomial if $\nu(C) \in \mathbb{R}[t]$ and $\nu(C) \neq 0$. It is called generic if mrpf $P=1$.

Motion polynomials form a subsemigroup of a special instance of the semigroup constructed in Equation (3). Hence, we may at least try to factor motion polynomials by means of Algorithm 2. For generic motion polynomials, which are exactly those motion polynomials that do not have real pseudofactors, it is guaranteed to work:

Lemma 2. Let $C=P+\varepsilon Q$ be a monic motion polynomial. If $M$ is a monic real quadratic factor of $\nu(C)$ but not a pseudofactor of $C$, then $\operatorname{czero}(C, M)$ is well defined (that is, $S:=\operatorname{lrem}(C, M)$ has a unique zero).

Proof. Because $M$ is not a pseudofactor of $C$ we have $\nu(S) \neq 0$. Similar to the proof of Lemma 1 we conclude that there exist dual number $c \in \mathbb{D}$ such that $\nu(S)=c M$ and $c \neq 0$. We claim that $c$ is invertible (in contrast to the proof of Lemma 1 this is not implied $\mathrm{b} c \neq 0$ ). Assume that $c$ is not invertible, that is, $c \in \varepsilon \mathbb{R}$. This implies that $\nu(S) \in \varepsilon \mathbb{R}[t]$ whence $M$ divides the primal part of $C$. But this is not possible because $M$ is assumed to be no pseudofactor of $C$. Hence $c \neq 0$ is invertible. It equals the norm of the leading coefficient of $S$ and this coefficient is invertible by a well-known property of dual numbers. Therefore, there exists a unique dual quaternion zero $h$ of $S$.

Corollary 2 ([3]). A generic motion polynomial $C=P+\varepsilon Q \in \mathbb{D} \mathbb{H}[t]$ with $P, Q \in \mathbb{H}[t]$ admits a factorization.

Proof. We recap the inductive proof of Theorem 3 and show that the necessary conclusion can be obtained for a generic motion polynomial $C$. Again the base case is obvious. Consider a quadratic real factor $M$ of $\nu(C)$. By assumption it is not a pseudofactor of $C$ whence $h:=\operatorname{czero}(C, M)$ is well defined by Lemma 2. The considerations at the beginning of Section 6.1 show that $t-h$ and $C^{\prime}:=\operatorname{lquo}(C, t-h)$ are motion polynomials. If $C^{\prime}$ was not generic, the same would be true for $C$, contrary to our assumption. Hence the induction hypothesis can be applied to $C^{\prime}$ and the proof is complete.

Remark 4. Algorithm 2 can be used to factor motion polynomials as long as czero $(C, M)$ is well-defined. In the generic case this is guaranteed.

Factorization results for non-generic motion polynomials and non-motion polynomials will be discussed later in Sections 6.3 and 6.5, respectively. We conclude this section with an example to demonstrate that success of Algorithm 2 for a split quaternion polynomial $C$ may depend on the order of quadratic factors of $\nu(C)$. 
Example 10. The polynomial

$$
\begin{aligned}
C=t^{4}-\left(\mathbf{i}_{s}-3 \mathbf{j}_{s}+2 \mathbf{k}_{s}+9\right) t^{3}+\left(7 \mathbf{i}_{s}-\right. & \left.12 \mathbf{j}_{s}+33 \mathbf{k}_{s}+43\right) t^{2} \\
& -\left(82 \mathbf{i}_{s}-59 \mathbf{j}_{s}+146 \mathbf{k}_{s}+38\right) t+162 \mathbf{i}_{s}-188 \mathbf{j}_{s}+213 \mathbf{k}_{s}-103
\end{aligned}
$$

admits the factorization $C=\left(t-h_{1}\right)\left(t-h_{2}\right)\left(t-h_{3}\right)\left(t-h_{4}\right)$ where

$$
\begin{gathered}
h_{1}=3 \mathbf{i}_{s}+\frac{21}{2} \mathbf{j}_{s}+\frac{23}{2} \mathbf{k}_{s}+2, \quad h_{2}=-\frac{91}{51} \mathbf{i}_{s}-\frac{2151}{221} \mathbf{j}_{s}-\frac{6791}{663} \mathbf{k}_{s}+2, \\
h_{3}=\frac{91}{51} \mathbf{i}_{s}-\frac{2667}{884} \mathbf{j}_{s}-\frac{6649}{2652} \mathbf{k}_{s}+2, \quad h_{4}=-2 \mathbf{i}_{s}-\frac{3}{4} \mathbf{j}_{s}+\frac{13}{4} \mathbf{k}_{s}+3
\end{gathered}
$$

This factorization can be computed by Algorithm 2. With

$$
M_{1}:=t^{2}-6 t+15, \quad M_{2}:=t^{2}-4 t-2, \quad M_{3}:=t^{2}-4 t+11, \quad M_{4}:=t^{2}-4 t+17
$$

we have $\nu(C)=M_{1} M_{2} M_{3} M_{4}$ and

$$
\begin{aligned}
& h_{4}=\operatorname{czero}\left(C, M_{1}\right), \\
& h_{3}=\operatorname{czero}\left(C^{\prime}, M_{2}\right) \quad \text { where } \quad C^{\prime}=\operatorname{lquo}\left(C, t-h_{4}\right), \\
& h_{2}=\operatorname{czero}\left(C^{\prime \prime}, M_{3}\right) \quad \text { where } \quad C^{\prime \prime}=\operatorname{lquo}\left(C^{\prime}, t-h_{3}\right), \\
& h_{1}=t-\operatorname{lquo}\left(C^{\prime \prime}, t-h_{2}\right) .
\end{aligned}
$$

A different order of quadratic factors may not work. With $k_{4}=-\mathbf{i}_{s}-\mathbf{j}_{s}+3 \mathbf{k}_{s}+2=\operatorname{czero}\left(C, M_{3}\right)$ we have

$$
\begin{aligned}
C^{\prime}:=\operatorname{lquo}\left(C, t-k_{4}\right) \\
\quad=t^{3}-\left(2 \mathbf{i}_{s}-2 \mathbf{j}_{s}-\mathbf{k}_{s}+7\right) t^{2}+\left(17 \mathbf{i}_{s}+4 \mathbf{j}_{s}+10 \mathbf{k}_{s}+26\right) t-52 \mathbf{i}_{s}-20 \mathbf{j}_{s}-35 \mathbf{k}_{s}-37
\end{aligned}
$$

but

$$
S:=\operatorname{lrem}\left(C^{\prime}, M_{1}\right)=\left(5 \mathbf{i}_{s}+16 \mathbf{j}_{s}+16 \mathbf{k}_{s}+5\right) t-22 \mathbf{i}_{s}-50 \mathbf{j}_{s}-50 \mathbf{k}_{s}-22
$$

and $\nu(S)=0$. Thus $M_{1}$ is a pseudofactor of $C^{\prime}$ but not of $C$. Algorithm 2 with this particular ordering of quadratic factors of $\nu(C)$ does not work.

6.2. Factorization of Quadratic Split Quaternion Polynomials. As demonstrated in Example 6, not all monic polynomials in $\mathbb{S}[t]$ admit factorizations. Here, we present a sufficient criterion for factorizability of quadratic polynomials in $\mathbb{S}[t]$. It relates existence of factorizations with the geometry of the projective space $P(\mathbb{S})$ over the vector space $\mathbb{S}$. Given a split quaternion $x \in \mathbb{S}$ we denote the corresponding point in $P(\mathbb{S})$ by $[x]$. Projective span is denoted by the symbol " $\vee$ ".

Definition 5. The quadric $\mathcal{N}$ in $P(\mathbb{S})$ given by the bilinear form $q: \mathbb{S} \times \mathbb{S} \rightarrow \mathbb{R},(x, y) \mapsto x y^{*}+y x^{*}$ is called the null quadric. A straight line contained in $\mathcal{N}$ is called a null line.

A point $[x]$ lies on the null quadric $\mathcal{N}$ if and only if $\nu(x)$ vanishes. It is easy to see (Lemma 4 below) that $\mathcal{N}$ is of hyperbolic type and contains two families of lines. In particular, null lines do exist.

Theorem 5. A quadratic polynomial $C=c_{2} t^{2}+c_{1} t+c_{0} \in \mathbb{S}[t]$ with invertible leading coefficient $c_{2}$ admits a factorization if the vectors $c_{0}, c_{1}$ and $c_{2}$ are linearly independent.

Lemma 3. The linear polynomial $S=s_{1} t+s_{0} \in \mathbb{S}[t]$ with linearly independent coefficients $s_{0}$ and $s_{1}$ satisfies $S S^{*}=0$ if and only if the straight line $\left[s_{0}\right] \vee\left[s_{1}\right]$ is a null line.

Proof. Because of $S S^{*}=s_{1} s_{1}^{*} t^{2}+\left(s_{1} s_{0}{ }^{*}+s_{0} s_{1}{ }^{*}\right) t+s_{0} s_{0}{ }^{*}$ we have $S S^{*}=0$ if and only if $q\left(s_{0}, s_{0}\right)=$ $q\left(s_{0}, s_{1}\right)=q\left(s_{1}, s_{1}\right)=0$. This is precisely the condition for the straight line $\left[s_{0}\right] \vee\left[s_{1}\right]$ to be contained in the quadric $\mathcal{N}$. 
Lemma 4. The quadric $\mathcal{N}$ contains two families of lines (the left and the right family) which are distinguished by the following property: For any two points $\left[p_{1}\right],\left[q_{1}\right]$ on a line of the left family, there exists $r_{1} \in \mathbb{S}$ such that $q_{1}=r_{1} p_{1}$. For any two points $\left[p_{2}\right],\left[q_{2}\right]$ on a line of the right family, there exists $r_{2} \in \mathbb{S}$ such that $q_{2}=p_{2} r_{2}$.

Proof. With $x=x_{0}+x_{1} \mathbf{i}_{s}+x_{2} \mathbf{j}_{s}+x_{3} \mathbf{k}_{s}$ we have $\frac{1}{2} q(x, x)=x_{0}^{2}-x_{1}^{2}-x_{2}^{2}+x_{3}^{2}$. Hence, the quadric $\mathcal{N}$ is of hyperbolic type and, indeed, carries two families of rulings. These are given as $[a] \vee[b]$ where

$$
a=1+\cos \varphi \mathbf{i}_{s}+\sin \varphi \mathbf{j}_{s}, \quad b=-\sin \varphi \mathbf{i}_{s}+\cos \varphi \mathbf{j}_{s}+e \mathbf{k}_{s},
$$

$\varphi \in[0,2 \pi)$ and $e=1$ or $e=-1$. Any point on $[c] \in[a] \vee[b]$ can be written as $c=\alpha a+\beta b$ and it suffices to discuss solvability of the equations $a x=c$ and $x a=c$. Since both equations are linear in the coefficients of $x$, a straight-forward calculation yields the solution

$$
x=\left(\alpha-x_{1} \cos \varphi-x_{2} \sin \varphi\right)+x_{1} \mathbf{i}_{s}+x_{2} \mathbf{j}_{s}+\left(\beta+x_{1} \sin \varphi-x_{2} \cos \varphi\right) \mathbf{k}_{s}
$$

of $\{e=1, x a=c\}$ and the solution

$$
x=\left(\alpha-x_{1} \cos \varphi-x_{2} \sin \varphi\right)+x_{1} \mathbf{i}_{s}+x_{2} \mathbf{j}_{s}-\left(\beta+x_{1} \sin \varphi-x_{2} \cos \varphi\right) \mathbf{k}_{s}
$$

of $\{e=-1, a x=c\}$. The systems $\{e=1, x a=c\}$ and $\{e=-1, a x=c\}$ have no solution.

Proof of Theorem 5. As usual, it is sufficient to prove the statement for monic polynomials, that is, $c_{2}=1$. We pick a monic quadratic factor $M_{1}$ of $\nu(C)$. The remainder polynomial $S_{1}:=\operatorname{lrem}\left(C, M_{1}\right)$ is (at most) of degree one and we can write $S_{1}=s_{1} t+s_{0}$. If $\nu\left(S_{1}\right) \neq 0, h:=\operatorname{czero}\left(C, M_{1}\right)$ is well defined by Lemma 1 . This means that one iteration of Algorithm 2 can be applied to $C$ to obtain a right factor $t-h$ and consequently a factorization, even if $\mathbb{S}$ is not a division ring. (The division ring property in the proof of Theorem 3 allows to conclude $\nu\left(S_{1}\right) \neq 0$ which is an assumption at this point.)

Next we consider the remaining case $\nu\left(S_{1}\right)=0$. If the coefficients $s_{1}$ and $s_{0}$ are linearly dependent, the coefficients of $C$ are linearly dependent as well. Hence, $s_{1}$ and $s_{0}$ are linearly independent and we may assume that $S_{1}$ parameterizes a null line. With $M_{2}:=M_{1}+S_{1}+S_{1}{ }^{*}$ and $S_{2}:=-S_{1}{ }^{*}$ we have $\nu(C)=M_{1} M_{2}$ and $C=M_{1}+S_{1}=M_{2}+S_{2}$. By Lemma $4, S_{1}$ or $S_{2}$ have a right zero. Lets assume, without loss of generality, that $S_{1}$ has this property. By a parameter transformation $t \mapsto t+u$ with a suitable $u \in \mathbb{R}$ we can ensure that $M_{1}$ is of the form $M_{1}=t^{2}+m$ with $m \in \mathbb{R}$. We have to show that there is a common right zero of $M_{1}$ and $S_{1}$. The right zeros of $S_{1}$ can be computed similarly as in the proof of Lemma 4: There exist $\alpha_{0}, \beta_{0}, \alpha_{1}, \beta_{1} \in \mathbb{R}$ such that

$$
s_{0}=\alpha_{0} a+\beta_{0} b \quad \text { and } \quad s_{1}=\alpha_{1} a+\beta_{1} b
$$

with $a, b$ as in (8) with $e=-1$. With $h=h_{0}+h_{1} \mathbf{i}+h_{2} \mathbf{j}+h_{3} \mathbf{k}$, the solution to $s_{1} h+s_{0}=0$ is given by (9)

$$
\begin{aligned}
& h_{0}=\frac{-1}{\alpha_{1}^{2}+\beta_{1}^{2}}\left(\alpha_{0} \alpha_{1}+\beta_{0} \beta_{1}+\left(\left(\alpha_{1}^{2}-\beta_{1}^{2}\right) \cos \varphi-2 \alpha_{1} \beta_{1} \sin \varphi\right) h_{1}+\left(2 \alpha_{1} \beta_{1} \cos \varphi+\left(\alpha_{1}^{2}-\beta_{1}^{2}\right) \sin \varphi\right) h_{2}\right), \\
& h_{3}=\frac{1}{\alpha_{1}^{2}+\beta_{1}^{2}}\left(\alpha_{1} \beta_{0}-\alpha_{0} \beta_{1}-\left(2 \alpha_{1} \beta_{1} \cos \varphi+\left(\alpha_{1}^{2}-\beta_{1}^{2}\right) \sin \varphi\right) h_{1}+\left(\left(\alpha_{1}^{2}-\beta_{1}^{2}\right) \cos \varphi-2 \alpha_{1} \beta_{1} \sin \varphi\right) h_{2}\right)
\end{aligned}
$$

with arbitrary real numbers $h_{1}, h_{2}$. A straightforward calculation shows that there is precisely one right zero of $M_{1}$ in this solution set. It is given by

$$
h=\frac{1}{2\left(\alpha_{0} \beta_{1}-\alpha_{1} \beta_{0}\right)}\left(h_{1} \mathbf{i}+h_{2} \mathbf{j}+h_{3} \mathbf{k}\right)
$$


where

$$
\begin{aligned}
& h_{1}=\left(\left(\alpha_{1}^{2}-\beta_{1}^{2}\right) m+\alpha_{0}^{2}-\beta_{0}^{2}\right) \sin \varphi+2\left(\alpha_{1} \beta_{1} m+\alpha_{0} \beta_{0}\right) \cos \varphi, \\
& h_{2}=\left(\left(\beta_{1}^{2}-\alpha_{1}^{2}\right) m-\alpha_{0}^{2}+\beta_{0}^{2}\right) \cos \varphi+2\left(\alpha_{1} \beta_{1} m+\alpha_{0} \beta_{0}\right) \sin \varphi, \\
& h_{3}=-\alpha_{0}^{2}-\beta_{0}^{2}-\left(\alpha_{1}^{2}+\beta_{1}^{2}\right) m .
\end{aligned}
$$

Note that the denominator of (10) does not vanish because otherwise the coefficients of $S_{1}$ and consequently also the coefficients of $C$ would be linearly dependent.

The quaternion $h$ is a common right zero of $M_{1}$ and $S_{1}$. By Theorem $2, t-h$ is a right factor of $M_{1}$ and $S_{1}$ and hence also of $C=M_{1}+S_{1}$. This implies existence of a factorization.

Example 11. We illustrate the "interesting" case in the proof of Theorem 5 by an example. Consider the polynomial $C=t^{2}+\left(1+\mathbf{i}_{s}\right) t+1+\mathbf{j}_{s}-\mathbf{k}_{s} \in \mathbb{S}[t]$. We have $C C^{*}=M_{1} M_{2}$ with $M_{1}=t^{2}+1$ and $M_{2}=(t+1)^{2}$. Polynomial division yields $C=M_{1}+S_{1}=M_{2}+S_{2}$ with

$$
S_{1}=\left(1+\mathbf{i}_{s}\right) t+\mathbf{j}_{s}-\mathbf{k}_{s} \text { and } S_{2}=\left(-1+\mathbf{i}_{s}\right) t+\mathbf{j}_{s}-\mathbf{k}_{s} .
$$

Note that $\nu\left(S_{1}\right)=\nu\left(S_{2}\right)=0$. The remainder $S_{2}$ has no right zeros, while the right zeros of $S_{1}$ are of the form

$$
h=-h_{1}+h_{1} \mathbf{i}_{s}+h_{2} \mathbf{j}_{s}+\left(1+h_{2}\right) \mathbf{k}_{s}, \quad h_{1}, h_{2} \in \mathbb{R} .
$$

The unique right zero $h=\mathbf{k}_{s}$ of $M_{1}$ among these solutions is obtained for $h_{1}=h_{2}=0$. Indeed, we have the factorization $C=\left(t+1+\mathbf{i}_{s}+\mathbf{k}_{s}\right)\left(t-\mathbf{k}_{s}\right)$.

6.3. Factorization of Non-Generic Motion Polynomials. We have already mentioned (and proved) the result of [3] on existence of factorizations of generic motion polynomials. These are polynomials $C=P+\varepsilon Q \in \mathbb{D} \mathbb{H}[t]$ with $P, Q \in \mathbb{H}[t]$ such that $\operatorname{mrpf} P=1$ and $\nu(C) \neq 0$. If $\operatorname{mrpf} P \neq 1$, general criteria on existence of factorizations are difficult to formulate. However, we would like to mention recent results by $[8,10]$ that ensure existence of factorizations for suitable multiples of not necessarily generic but bounded motion polynomials.

Definition 6. A motion polynomial $C=P+\varepsilon Q$ with $P, Q \in \mathbb{H}[t]$ is called bounded if mrpf $P$ has no real zeros and unbounded otherwise.

The name "bounded" comes from the fact that all trajectories of a bounded motion polynomials are bounded rational curves.

Theorem 6 ([8, 10]). Consider a bounded monic motion polynomial $C=P+\varepsilon Q \in \mathbb{D} \mathbb{H}[t]$ with $P$, $Q \in \mathbb{H}[t]$.

- There exists a polynomial $S \in \mathbb{R}[t]$ of degree $\operatorname{deg} S \leq \operatorname{deg} \operatorname{mrpf} P$ such that $C S$ admits a factorization.

- If $\operatorname{gcd}(P, \nu(Q))=1$ there exists a polynomial $D \in \mathbb{H}[t]$ of degree $\operatorname{deg} D=\frac{1}{2} \operatorname{deg} \operatorname{mrpf} P$ such that $C D$ admits a factorization.

The algorithm of [10] for computing the co-factor $D$ is too complicated to be discussed here. We confine ourselves to a simple example and remark that some aspects of this factorization algorithm are used in our proof of Theorem 7 below.

Example 12. Consider the polynomial $C=t^{2}+1+\varepsilon \mathbf{i}$. As mentioned in Example 9, it admits no factorization with motion polynomial factors. But with $S=t^{2}+1$ and $D=t-\mathbf{k}$ we have

$$
\begin{gathered}
C S=\left(t+\frac{3}{5} \mathbf{j}-\frac{4}{5} \mathbf{k}\right)\left(t-\frac{3}{5} \mathbf{j}+\frac{4}{5} \mathbf{k}+\varepsilon\left(\frac{2}{5} \mathbf{j}+\frac{3}{10} \mathbf{k}\right)\right)\left(t-\frac{3}{5} \mathbf{j}+\frac{4}{5} \mathbf{k}-\varepsilon\left(\frac{2}{5} \mathbf{j}+\frac{3}{10} \mathbf{k}\right)\right)\left(t+\frac{3}{5} \mathbf{j}-\frac{4}{5} \mathbf{k}\right), \\
C D=(t+\mathbf{k})\left(t-\mathbf{k}-\frac{1}{2} \varepsilon \mathbf{j}\right)\left(t-\mathbf{k}+\frac{1}{2} \varepsilon \mathbf{j}\right) .
\end{gathered}
$$


Above results state that existence of a motion polynomial factorization can be guaranteed after multiplication with a real polynomial (which does not change the underlying motion) or with a quaternion polynomial (which does not change the trajectory of the origin). In [7] and [8] this was used for the construction of linkages with a prescribed bounded rational trajectory (Figure 3 ).

6.4. Factorization of Unbounded Motion Polynomials. If $C$ is an unbounded motion polynomial, existence of a factorization is not guaranteed, not even after multiplication with a real polynomial $S \in \mathbb{R}[t]$ or a quaternion polynomial $D \in \mathbb{H}[t]$. Depending on the application one has in mind, it might be possible to transform an unbounded motion polynomial into a bounded motion polynomial. We may, for example substitute a rational expression $A / B$ with $A, B \in \mathbb{R}[t]$ for the indeterminate $t$ in $C$ and try to factor $B^{\operatorname{deg} C} C(A / B)$ instead. This amounts to a not necessarily invertible re-parameterization of the motion. In particular, it is possible to parameterize only one part of the original motion and transform $C$ to a bounded motion polynomial.

However, there is a dense set of unbounded motions polynomials that admit a factorization:

Theorem 7. If an unbounded motion polynomial $C=P+\varepsilon Q \in \mathbb{D} \mathbb{H}[t]$ with $P, Q \in \mathbb{H}[t]$ is such that all linear real factors of mrpf $P$ have multiplicity one, there exists a real polynomial $D \in \mathbb{R}[t]$ such that $C D$ admits a factorization with linear motion polynomial factors.

Proof. We set $p^{\prime}:=\operatorname{mrpf} P$ and denote by

$$
p=\prod_{i=1}^{n}\left(t-a_{i}\right), \quad a_{1}, a_{2}, \ldots, a_{n} \in \mathbb{R} .
$$

the product of all monic linear real factors of $p^{\prime}$. We then have $C C^{*}=p^{2} U$ and $U \in \mathbb{R}[t]$ has only irreducible quadratic real factors.

We pick one linear factor of $p$, say $t-a_{1}$, and set $M:=\left(t-a_{1}\right)^{2}$. Because mrpf $P$ has no linear real polynomial factor of multiplicity two, $M$ is not a pseudofactor of $C$ and Lemma 2 can be applied to compute $h:=\operatorname{czero}(C, M)$. We now have $C=\tilde{C}(t-h)$ for some motion polynomial $\tilde{C}$ which is amenable to one further iteration of above construction (which is essentially one iteration of Algorithm 2). Treating all linear real factors of $p$ in like manner, we obtain a polynomial $H \in \mathbb{D} \mathbb{H}[t]$ that admits a factorization with motion polynomial factors such that $C=C^{\prime} H$ and $C^{\prime}=P^{\prime}+\varepsilon Q^{\prime}$ is bounded, that is, mrpf $P^{\prime}$ has no linear real factor. For bounded motion polynomials the statement is known to be true [10, Theorem 1].

There exist unbounded motion polynomials $C$ such that $C D$ does not admit a factorization for all $D \in \mathbb{H}[t]$ (and in particular for real polynomials):

Example 13. Consider the unbounded motion polynomial $C=\left(t-a_{0}\right)\left(t-a_{1}\right)+\varepsilon \mathbf{i}$ with $a_{0}=a_{1}=0$ and a quaternion polynomial $D \in \mathbb{H}[t]$ with factorization $D=\prod_{i=2}^{n}\left(t-a_{i}\right)$ where $a_{2}, a_{3}, \ldots, a_{m} \in \mathbb{H}$. Then, the primal part of the product $C D$ has the factorization $\prod_{i=0}^{n}\left(t-a_{i}\right)$ and a suitable dual part exists if the system

$$
\mathbf{i} D=\sum_{i=0}^{n}\left(\prod_{j=0, j \neq i}^{n}\left(t-a_{j}\right)\right) b_{i}
$$

has a solution for $b_{0}, b_{1}, \ldots, b_{n}$. But this is not possible because the multiplicity of the factor $t$ on the right-hand side is always strictly larger than the multiplicity of this factor on the left-hand side.

6.5. Factorization by Projection. We conclude this text with a factorization technique applicable to non-motion polynomials in $\mathbb{D H}$. Here, Algorithm 2 fails already at an early stage because the norm polynomial $\nu(C)$ is no longer real. More generally, consider the Clifford algebra $C \ell_{(p, q, 1)}$ and denote the basis elements of $\mathbb{R}^{n}$ that square to \pm 1 by $e_{1}, e_{2}, \ldots, e_{m}$ where $m=p+q$. There are $n=2^{m}-1$ 
generators of $C \ell_{(p, q, 1)}$ that are products of above basis elements with non-zero square. We denote them by $\mathbf{i}_{1}, \mathbf{i}_{2}, \ldots, \mathbf{i}_{n}$ and we write $\varepsilon$ for the generator that squares to zero. Note that the real unit $1=e_{0}$ has non-zero square as well.

Every element $c \in C \ell_{(p, q, 1)}$ can be uniquely written as $c=a+b$ where $a \in\left\langle 1, \mathbf{i}_{1}, \mathbf{i}_{2}, \ldots, \mathbf{i}_{n}\right\rangle$ and $b \in\left\langle\varepsilon, \mathbf{i}_{1} \varepsilon, \mathbf{i}_{2} \varepsilon, \ldots, \mathbf{i}_{n} \varepsilon\right\rangle$. In the context of dual quaternions, $a$ is called the primal part and $b$ is called the dual part and we use these notions here as well. A polynomial $C \in C \ell_{(p, q, 1)}$ has a unique representation as $C=A+B$ where $A$ is a polynomial whose coefficients have zero dual part and $B$ is a polynomial whose coefficients have zero primal part. We call $A$ and $B$, primal part and dual part, respectively, of $C$.

Assume now that the primal part of the monic polynomial $C$ admits a factorization in $C \ell_{(p, q, 0)}$, that is, $A=\left(t-a_{1}\right)\left(t-a_{2}\right) \cdots\left(t-a_{n}\right)$ with $a_{1}, a_{2}, \ldots, a_{n} \in C \ell_{(p, q, 0)}$. We make the ansatz

$$
C=\left(t-a_{1}-b_{1}\right)\left(t-a_{2}-b_{2}\right) \cdots\left(t-a_{n}-b_{n}\right)
$$

with yet undetermined coefficients $b_{1}, b_{2}, \ldots, b_{n}$ of vanishing primal part. Comparing coefficients on both sides of (11) yields a system of linear equations for the unknown real coefficients of $b_{1}, b_{2}, \ldots, b_{n}$. The number of equations and the number of unknowns both equal $(m+1) n$. Thus we can state:

If the primal part of a monic polynomial $C \in C \ell_{(p, q, 1)}$ admits a factorization, a factorization of $C$ exists if the system of $(m+1) n$ linear equations in the same number of unknowns arising from comparing coefficients of (11) has solutions.

Generically, the solution to the linear system is unique but we already encountered cases with infinitely many solutions or with no solution at all (Examples 7 and 8). The algebra and geometry of factorization of non-motion polynomials in $\mathbb{D H}[t]$ (and in particular a kinematic interpretation) occurs in the theses $[9,11]$ but numerous open issues remain. In particular, sufficient criteria for existence of factorizations, that is, solvability of the system of linear equations arising from (11), would be desirable. While the factorization of motion polynomials gives rise to a decomposition of rational motions into a sequence of rotations, factorization of non-motion polynomials in $\mathbb{D} \mathbb{H}[t]$ has in interpretation as decomposition into so-called vertical Darboux motions [11].

\section{ACKNOWLEDGMENT}

Daniel Scharler and Hans-Peter Schröcker were supported by the Austrian Science Fund (FWF): P 31061 (The Algebra of Motions in 3-Space). The authors gratefully acknowledge useful comments and suggestions by anonymous reviewers that helped to improve this text. All authors thank the Erwin Schrödinger International Institute for Mathematics and Physics for its hospitality when completing the last revision of this paper.

\section{REFERENCES}

[1] I. Niven, Equations in quaternions, Amer. Math. Monthly 48 (10) (1941) 654-661.

[2] B. Gordon, T. S. Motzkin, On the zeros of polynomials over division rings, Trans. Amer. Math. Soc. 116 (1965) $218-226$.

[3] G. Hegedüs, J. Schicho, H.-P. Schröcker, Factorization of rational curves in the Study quadric and revolute linkages, Mech. Machine Theory 69 (1) (2013) 142-152. arXiv:1202.0139.

[4] Z. Li, T.-D. Rad, J. Schicho, H.-P. Schröcker, Factorization of rational motions: A survey with examples and applications, in: S.-H. Chang (Ed.), Proceedings of the 14th IFToMM World Congress, 2015.

[5] Z. Li, J. Schicho, H.-P. Schröcker, 7R Darboux linkages by factorization of motion polynomials, in: S.-H. Chang (Ed.), Proceedings of the 14th IFToMM World Congress, 2015.

[6] Z. Li, J. Schicho, H.-P. Schröcker, Spatial straight-line linkages by factorization of motion polynomials, J. Mechanisms Robotics 8 (2) (2016) 021002.

[7] M. Gallet, C. Koutschan, Z. Li, G. Regensburger, J. Schicho, N. Villamizar, Planar linkages following a prescribed motion, Math. Comp. 87 (2017) 473-506.

[8] Z. Li, J. Schicho, H.-P. Schröcker, Kempe's universality theorem for rational space curves, Found. Comput. Math.arXiv: 1509.08690. 
[9] D. Scharler, Characterization of lines in the extended kinematic image space, Master thesis, University of Innsbruck (2017).

[10] Z. Li, J. Schicho, H.-P. Schröcker, Factorization of motion polynomials, Accepted for publication in J. Symbolic Comp. (2018). arXiv: 1502.07600.

[11] T.-D. Rad, Factorization of motion polynomials and its application in mechanism science, Phd thesis, University of Innsbruck (2018).

[12] O. Ore, Theory of non-commutative polynomials, Annh. of Math. (2) 34 (3) (1933) 480-508.

[13] R. S. Palais, The classification of real division algebras, Amer. Math. Monthly 75 (4) (1968) 366-368.

[14] R. M. Corless, S. M. Watt, L. Zhi, QR factoring to compute the GCD of univariate approximate polynomials, IEEE Trans. Signal Process. 52 (12) (2004) 3394-3402.

[15] E. Kaltofen, Z. Yang, L. Zhi, Approximate greatest common divisors of several polynomials with linearly constrained coefficients and singular polynomials, in: J.-G. Dumas (Ed.), Proceedings of the 2006 International Symposium on Symbolic and Algebraic Computation, ISSAC '06, ACM, Genoa, Italy, 2006, pp. 169-176. doi:10.1145/1145768. 1145799.

[16] D. Bini, V. Y. Pan, Polynomial and Matrix Computations: Fundamental Algorithms, Springer Science \& Business Media, 2012.

[17] V. Y. Pan, Computation of approximate polynomial GCDs and an extension, Inform. and Comput. 167 (2) (2001) $71-85$.

[18] Z. Li, Z. Yang, L. Zhi, Blind image deconvolution via fast approximate GCD, in: Proceedings of the 2010 International Symposium on Symbolic and Algebraic Computation, ACM, 2010, pp. 155-162.

[19] D. Klawitter, Clifford Algebras. Geometric Modelling and Chain Geometries with Application in Kinematics, Springer Spektrum, 2015.

[20] J. Selig, Geometric Fundamentals of Robotics, 2nd Edition, Monographs in Computer Science, Springer, 2005.

[21] H. Pottmann, J. Wallner, Computational Line Geometry, Mathematics and Visualization, Springer, 2010, 2nd printing.

[22] N. Wildberger, Universal hyperbolic geometry I: Trigonometry, Geom. Dedicata 163 (2013) 215-274.

[23] Z. Li, J. Schicho, H.-P. Schröcker, The geometry of quadratic quaternion polynomials in Euclidean and non-Euclidean planes, in: L. Cocchiarella (Ed.), ICGG 2018 - Proceedings of the 18th International Conference on Geometry and Graphics, Springer International Publishing, Cham, 2019, pp. 298-309. arXiv:1805.03539.

[24] G. Hegedüs, J. Schicho, H.-P. Schröcker, Four-pose synthesis of angle-symmetric 6R linkages, J. Mechanisms Robotics 7 (4).

(Zijia Li) Institute of Discrete Mathematics and Geometry, Vienna University of Technology, Wiedner Hauptstrasse 8-10/104, 1040 Vienna, Austria

E-mail address: zijia.li@tuwien.ac.at

(Daniel Scharler, Hans-Peter Schröcker) Unit Geometry and CAD, University of Innsbruck, Technikerstr. 13, 6020 Innsbruck, Austria

$U R L:$ https://geometrie.uibk.ac.at/

E-mail address: \{daniel.scharler\}\{hans-peter.schroecker\}@uibk.ac.at 